\title{
THERMODYNAMIC PROPERTIES OF $D$-WAVE PAIRED SUPERCONDUCTORS IN THE LOW TEMPERATURE AND SUBCRITICAL REGIONS
}

\author{
W. Kumala, R. Gonczarek and M. Mulak \\ Institute of Physics, Wrocław University of Technology \\ Wybrzeże Wyspiańskiego 27, 50-370 Wrocław, Poland
}

(Received May 11, 1999; revised version October 13, 1999; in final form February 11, 2000)

$D$-wave paired isotropic Fermi system in the limiting cases $T \rightarrow T_{\mathrm{c}}$ and $T \rightarrow 0$ is considered. Values of the energy gap, the thermodynamic potential, and the specific heat as a function of temperature are given for admissible solutions and compared with values for $S$-wave paired system. It is shown that the linear term of low temperature specific heat vanishes likewise in case of $S$-wave pairing. The obtained results make prospects to define the geometry of the energy gap in virtue of the behavior of the specific heat in the limiting temperature cases for heavy-fermion superconductors.

PACS numbers: $74.20 .-\mathrm{z}$

\section{Introduction}

The Fermi-liquid theory of superconductivity can be applied to the broad range of systems of the new generation superconductors, i.e. heavy-fermion, cuprates, and organic superconductors which reveal the existence of the unconventional Cooper pairs [1, 2]. Taking into account the energy gap equation obtained applying self-consistent Green function in the framework of the weak coupling approximation, one can easy introduce to the formalism the anisotropic pairing interaction [3], particle-hole asymmetry on the Fermi level [4], as well as additional corrections of the Fermi-liquid interaction arising from the Hubbard type approaches [5].

In this paper we study the thermodynamic properties of isotropic, $D$-wave paired Fermi system in the limiting temperature cases, which are proved to be quite different than typical properties of $S$-wave paired system [6]. For that purpose we expand the value of energy gap in power series of temperature for admissible energy gap shapes and then use relation between the functions of energy gap and thermodynamic potential. 


\section{General relations}

The BCS gap equation can be written as follows [7]:

$$
\Delta_{k}=-(1 / 2) \sum_{\boldsymbol{k}^{\prime}} V_{\boldsymbol{k} \boldsymbol{k}^{\prime}} \Delta_{\boldsymbol{k}^{\prime}} E_{\boldsymbol{k}^{\prime}}^{-1} \tanh \left(E_{\boldsymbol{k}^{\prime}} / 2 k_{\mathrm{B}} T\right),
$$

where $E_{k}^{2}=\xi_{k}+\Delta_{k}^{+} \Delta_{k}$ is the quasiparticle excitation energy, $\xi_{k}$ is the single-particle energy relative to the Fermi energy and $\Delta_{k}$ is the momentum-dependent energy gap. The formalism of BCS weak-coupling theory require to the interaction potential $V_{\boldsymbol{k} \boldsymbol{k}^{\prime}}$ has significant impact only on particles in the vicinity of the Fermi surface so one can take into account only this region. If the system is rotationally symmetric $V_{\boldsymbol{k}^{\prime}}$ may be expanded in terms of the Legendre polynomials $P_{L}\left(\boldsymbol{k} \cdot \boldsymbol{k}^{\prime}\right)$ or spherical harmonics $Y_{L M}(k)$

$$
V_{\boldsymbol{k} \boldsymbol{k}^{\prime}}=\sum_{L} V_{L} P_{L}\left(k \cdot k^{\prime}\right)=\sum_{L M}\left[V_{L} /(2 L+1)\right] Y_{L M}^{*}(k) Y_{L M}\left(k^{\prime}\right),
$$

where $k, k^{\prime}$ are the versors denoting the direction of the quasiparticles momentum on the Fermi surface, $V_{L}$ are coefficients of expansion of the pairing potential in series of the Legendre polynomials and $-L \leq M \leq L$.

Replacing the summation over momentum space by integration over spherical angle $\Omega_{\boldsymbol{k}^{\prime}}$, Eq. (1) reduces to the form

$$
\Delta_{k}=\sum_{L M} g_{L} Y_{L M}(k)\left\langle Y_{L M}^{*}\left(k^{\prime}\right) \Delta_{k^{\prime}} \Phi_{k^{\prime}}\right\rangle
$$

where $g_{L}=-V_{L} N(0) /(2 L+1)$ are dimensionless coupling parameters, $N(0)$ denotes the density of states per spin and unit volume on the Fermi level,

$$
\Phi_{k^{\prime}}=\int_{0}^{\hbar \omega} E_{k^{\prime}}^{-1} \tanh \left(E_{k^{\prime}} / 2 k_{\mathrm{B}} T\right) \mathrm{d} \xi
$$

$\hbar \omega$ is the cut-off energy and the following notation is used:

$$
\langle\ldots\rangle=\int \mathrm{d} \Omega_{k^{\prime}} / 4 \pi \cdot \ldots
$$

It is useful to split $\Delta_{k}$ into two parts, first $\Delta$ characterizing the average modulus of energy gap, second $D_{k}$ defining the spherical angles dependence, which can be additionally expanded in terms of the spherical harmonics

$$
\Delta_{k}=\Delta \cdot D_{k}=\Delta \cdot \sum_{L M} \Delta_{L M} Y_{L M}(k),
$$

where $\Delta_{L M}$ are coefficients of expansion of $D_{k}$ in series of spherical harmonics and the following relation is fulfilled:

$$
\left\langle\left|\Delta_{k}\right|^{2}\right\rangle=\Delta^{2}\left\langle\left|D_{k}\right|^{2}\right\rangle=\Delta^{2} \sum_{L M}\left|\Delta_{L M}\right|^{2}=\Delta^{2} .
$$

Now Eq. (3) can be separated into the system of equations

$$
\Delta_{L M}=g_{L}\left\langle D_{\boldsymbol{k}} Y_{L M}^{*}(k) \Phi_{\boldsymbol{k}}\right\rangle .
$$

Note that if $g_{L^{\prime}}=0$ all $\Delta_{L^{\prime} M}$ must be equal to zero; on the other hand, $\Delta_{L M}=0$ satisfy Eq. (8) for arbitrary value of coupling parameter and temperature. 
After integrating $\Phi_{k}$ by parts

$$
\begin{aligned}
\Phi_{\boldsymbol{k}}= & \int_{0}^{\hbar \omega / 2 k_{\mathrm{B}} T}\left(\sqrt{u^{2}+\tau^{2}\left|D_{\boldsymbol{k}}\right|^{2}}\right)^{-1} \tanh \sqrt{u^{2}+\tau^{2}\left|D_{k}\right|^{2}} \mathrm{~d} u \\
= & \ln \frac{2 \hbar \omega}{2 k_{\mathrm{B}} T}-\ln \left(\tau\left|D_{\boldsymbol{k}}\right|\right) \tanh \left(\tau\left|D_{k}\right|\right)-\int_{0}^{\infty}\left(u+\sqrt{u^{2}+\tau^{2}\left|D_{\boldsymbol{k}}\right|^{2}}\right) \\
& \times \frac{\partial}{\partial u} \tanh \sqrt{u^{2}+\tau^{2}\left|D_{\boldsymbol{k}}\right|^{2}} \mathrm{~d} u=\ln \frac{2 \hbar \omega}{2 k_{\mathrm{B}} T}-W\left(\tau\left|D_{\boldsymbol{k}}\right|\right),
\end{aligned}
$$

where $\tau=\Delta / 2 k_{\mathrm{B}} T, u=\xi / 2 k_{\mathrm{B}} T$. The upper limit in the integral is extended up to infinity because of quick convergence of integrals. $W(x)$ is an analytic function, which for small arguments may be approximated by polynomial

$$
W(x) \approx \ln \frac{2 \mathrm{e}^{\gamma}}{\pi}+\frac{7 \zeta(3)}{2 \pi^{2}} x^{2}+\ldots,
$$

with $\gamma \approx 0.5772$ as the Euler constant and $\zeta(3) \approx 1.202$ as the zeta Riemann function; whereas for enough large arguments (i.e. $x>5$ ) it increases logarithmically

$$
W(x) \approx \ln (x) .
$$

For $T=T_{c}$ all coefficients $\Delta_{L M}$ approach zero and Eqs. (8) reduce to one equation independent of $D_{k}$ :

$$
\frac{1}{g_{\max }}=\ln \frac{2 \hbar \omega}{2 k_{\mathrm{B}} T_{\mathrm{c}}}-\ln \frac{\pi}{2 \mathrm{e}^{\gamma}},
$$

where $g_{\max }$ is the highest coupling parameter and $\gamma \approx 0.5772$ is the Euler constant. This means that all the admissible solutions will have the same critical temperature $T_{\mathrm{c}}$ dependent only on highest coupling parameter.

Substituting (9) into Eqs. (8) and using (12) to eliminate $\hbar \omega$, one gets parametric equations for value of reduced average energy gap and reduced temperature

$$
\begin{aligned}
& \frac{T}{T_{\mathrm{c}}}=\frac{\pi}{2 \mathrm{e}^{\gamma}} \exp \left(\frac{1}{g_{\max }}-\frac{1}{g_{L}}\right) \exp \left[-\left\langle\frac{D_{k} Y_{L M}^{*}(k)}{\Delta_{L M}} W\left(\tau\left|D_{k}\right|\right)\right\rangle\right], \\
& \frac{\Delta}{2 k_{\mathrm{B}} T_{\mathrm{c}}}=2 \frac{\Delta}{2 k_{\mathrm{B}} T} \frac{T}{T_{\mathrm{c}}}=2 \tau \frac{T}{T_{\mathrm{c}}} .
\end{aligned}
$$

For $T=0$ the parameter $\tau$ approaches infinity, so substituting (11) into (13) one can get formula for $\Delta_{0} / k_{\mathrm{B}} T_{\mathrm{c}}$, where $\Delta_{0} \equiv \Delta(T=0)$.

In the case of $D$-wave pairing the only coupling parameter that does not vanish is $g_{2}$. The possible ground state in $D$-wave paired isotropic Fermi system has been theoretically examined by Anderson and Morel [8]. They have found five particular solutions, which fulfilled Eqs. (8) for the zero temperature and to which can be brought the other solutions by the rotation of coordinate system, with

$$
D_{k}=\left\{\begin{array}{l}
Y_{20}(k) \\
Y_{2 \pm 1}(k) \\
Y_{2 \pm 2}(k) \\
(1 / \sqrt{2}) Y_{20}(k)+(1 / 2)\left[Y_{22}(k)-Y_{2-2}(k)\right]=d_{x y z}(k) \\
(1 / \sqrt{2})\left[Y_{22}(k)+Y_{2-2}(k)\right]=d_{x y}(k),
\end{array}\right.
$$


and $\Delta_{0} / k_{\mathrm{B}} T_{\mathrm{c}}$ ratio equal to: $1.381,1.543,1.543,1.578,1.323$, respectively (for $S$-wave pairing $D_{k}=Y_{00}(k)=1$ and $\left.\Delta_{0} / k_{\mathrm{B}} T_{\mathrm{c}}=1.764\right)$. Fortunately, in these cases, $D_{\boldsymbol{k}}$ is preserved for arbitrary temperature, and only the value of $\Delta$ changes. If the admissible energy gap shape is known and there exist only one non-zero coupling parameter, Eqs. (8) can be reduced to one equation multiplying their sides by $\Delta_{L M}^{*}$ and summing them up

$$
1 / g_{L}=\left\langle\left|D_{\boldsymbol{k}}\right|^{2} \Phi_{\boldsymbol{k}}\right\rangle \text {. }
$$

Analogously Eqs. (13) reduce to the form

$$
\frac{T}{T_{\mathrm{c}}}=\frac{\pi}{2 \mathrm{e}^{\gamma}} \exp \left[-\left\langle\left|D_{\boldsymbol{k}}\right|^{2} W\left(\tau\left|D_{k}\right|\right)\right\rangle\right] .
$$

Solving numerically Eq. (17) and using (14), with $\tau$ running from zero to infinity, the plot of $\Delta / k_{\mathrm{B}} T_{\mathrm{c}}$ as a function of $T / T_{\mathrm{c}}$ may be obtained (see Fig. 2a).

\section{Energy gap in limiting cases $T \rightarrow T_{\mathrm{c}}$ and $T \rightarrow 0$}

For $T_{\mathrm{c}}-T \ll T_{\mathrm{c}}$ and $T \ll T_{\mathrm{c}}$ the function of the energy gap can be expanded in power series of the parameter $\left(1-T / T_{\mathrm{c}}\right)$ or $T / T_{\mathrm{c}}$, respectively. For a temperature close to $T_{\mathrm{c}}$, the parameter $\tau$ approaches zero so $\tau\left|D_{\boldsymbol{k}}\right|$ is small for each $k$. Substituting (10) into (17) one gets

$$
\frac{\Delta}{k_{\mathrm{B}} T_{\mathrm{c}}}=\left[8 \pi^{2} /\left(7 \zeta(3)\left\langle\left|D_{k}\right|^{4}\right\rangle\right)\right]^{1 / 2}\left(1-\frac{T}{T_{\mathrm{c}}}\right)^{1 / 2}
$$

where only terms up to $\left(1-T / T_{\mathrm{c}}\right)$ were taken into account. The value of $\left\langle\left|D_{k}\right|^{4}\right\rangle$ is equal to 2.143 for $Y_{20}, d_{x y}$ solutions, 1.429 for $Y_{2 \pm 1}, Y_{2 \pm 2}, d_{x y z}$ ones, and 1 for $S$-wave pairing case, respectively.

The calculation of $\left\langle\left|D_{\boldsymbol{k}}\right|^{2} W\left(\tau\left|D_{\boldsymbol{k}}\right|\right)\right\rangle$ in the low temperature limit is more difficult because in this case $\tau\left|D_{\boldsymbol{k}}\right|$, for different $k$, takes values from a wide interval.

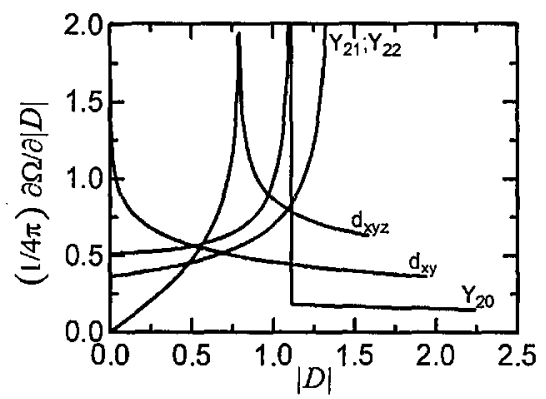

Fig. 1. The normalized to one density of states on Fermi surface as a function of reduced energy gap $|D|$, for different shapes of $D$-wave energy gap. For small $|D|$ in the case of $Y_{20}, Y_{21}, Y_{22}$ harmonics, the reduced density of states $(1 / 4 \pi) \partial \Omega / \partial|D|$ is roughly constant, whereas for $d_{x y z}$ (the most symmetrical $D$-wave solution) the linear dependence dominates, and for $d_{x y}$ a logarithmic function is observed. In $S$-wave pairing case, the energy gap is constant over all Fermi surface, so $(1 / 4 \pi) \partial \Omega / \partial|D|=\delta(|D|-1)$, where $\delta(x)$ is the Dirac delta function. 
It is very useful to replace integration over angle $\Omega_{k}$ by integration over the value of $\left|D_{k}\right|$ :

$$
\begin{aligned}
\langle\ldots\rangle & =\int \frac{\mathrm{d} \Omega_{k}}{4 \pi} \ldots=\int_{0}^{|D|_{\max }} \mathrm{d}|D| \frac{1}{4 \pi} \frac{\partial \Omega}{\partial|D|} \cdot \ldots \\
& =\int_{0}^{|D|_{\max }} \mathrm{d}|D| f(|D|) \cdot \ldots,
\end{aligned}
$$

where $|D|_{\max }$ is the maximum value of $\left|D_{\boldsymbol{k}}\right|$. In Fig. 1 functions $f(|D|)=$ $(1 / 4 \pi) \partial \Omega / \partial|D|$ for all shapes are depicted. Note that $f(|D|)$ is identical for solutions with $D_{k}=Y_{2 \pm 1}(k)$ and $D_{k}=Y_{2 \pm 2}(k)$. This causes that in isotropic system all properties for these solutions will be the same. Based on the behavior of $W(x)$ (see (11)), one may separate a part of integral in which $W(\tau|D|)$ increases non-logarithmically i.e. with $|D|<5 / \tau$ :

$$
\begin{aligned}
& \left\langle\left|D_{k}\right|^{2} W\left(\tau\left|D_{k}\right|\right)\right\rangle=\int_{0}^{|D|_{\max }}|D|^{2} W(\tau|D|) f(|D|) \mathrm{d}|D| \\
& \quad \approx \int_{0}^{5 / \tau}|D|^{2}[W(\tau|D|)-\ln (\tau|D|)] f(|D|) \mathrm{d}|D| \\
& \quad+\int_{0}^{|D|_{\max }}|D|^{2} \ln (\tau|D|) f(|D|) \mathrm{d}|D|
\end{aligned}
$$

Because $\tau$ is large ( $\tau=\Delta / 2 k_{\mathrm{B}} T$, and temperature tends to zero) the upper limit in the first integral in Eq. (20) is small and one can approximate $f(|D|)$ by using polynomial

$$
\sum_{n} A_{n}|D|^{n}= \begin{cases}2 / \sqrt{15}+(1 / \sqrt{15})|D|^{2} & \text { for } Y_{20} \\ \sqrt{2 / 15}+(1 / 15)|D| & \text { for } Y_{21} \text { or } Y_{22} \\ (4 / 5)|D|+|D|^{3} & \text { for } d_{x y z},\end{cases}
$$

or logarithmic function

$$
E \ln (|D|)+F=-0.1645 \ln (|D|)+0.4504 \text { for } d_{x y} .
$$

In the first case of approximation

$$
\begin{aligned}
\int_{0}^{5 / \tau} & |D|^{2}[W(\tau|D|)-\ln (\tau|D|)] \sum_{n} A_{n}|D|^{n} \mathrm{~d}|D| \\
\quad= & \sum_{n} \frac{A_{n}}{\tau^{n+3}} \int_{0}^{5} x^{n+2}[W(x)-\ln (x)] \mathrm{d} x=\sum_{n} \frac{A_{n} C_{n}}{\tau^{n+3}},
\end{aligned}
$$

and in the second one respectively

$$
\begin{aligned}
& \int_{0}^{5 / \tau}|D|^{2}[W(\tau|D|)-\ln (\tau|D|)][E \ln (|D|)+F] \mathrm{d}|D| \\
& =\frac{F-E \ln (\tau)}{\tau^{3}} \int_{0}^{5} x^{2}[W(x)-\ln (x)] \mathrm{d} x+\frac{E}{\tau^{3}} \int_{0}^{5} x^{2} \ln (x)[W(x)-\ln (x)] \mathrm{d} x \\
& =\frac{[F-E \ln (\tau)] C_{0}}{\tau^{3}}+\frac{E G}{\tau^{3}}
\end{aligned}
$$


where $C_{n}=\int_{0}^{5} x^{n+2}[W(x)-\ln (x)] \mathrm{d} x$, hence $C_{0}=0.3535, C_{1}=0.4706, C_{2}=$ $0.8428, C_{3}=1.879 ; G=\int_{0}^{5} x^{2} \ln (x)[W(x)-\ln (x)] \mathrm{d} x=0.0321$. Moreover, calculating the last integral in (20) using (7) and (17) with (11) one gets

$$
\begin{aligned}
& \int_{0}^{|D|_{\max }}|D|^{2} \ln (\tau|D|) f(|D|) \mathrm{d}|D|=\ln (\tau)\left\langle\left|D_{\boldsymbol{k}}\right|^{2}\right\rangle+\left\langle\left|D_{k}\right|^{2} \ln \left(\left|D_{\boldsymbol{k}}\right|\right)\right\rangle \\
& \quad=\ln (\tau)+\ln \left[\left(\pi / 2 \mathrm{e}^{\gamma}\right) \cdot\left(2 k_{\mathrm{B}} T_{\mathrm{c}} / \Delta_{0}\right)\right] .
\end{aligned}
$$

Substituting above results into Eq. $(20)$ and using formula (17), $\exp (-x) \approx 1-x$, and $\tau \approx \Delta_{0} / 2 k_{\mathrm{B}} T$, one has

$$
\begin{aligned}
& \Delta / \Delta_{0}
\end{aligned}
$$

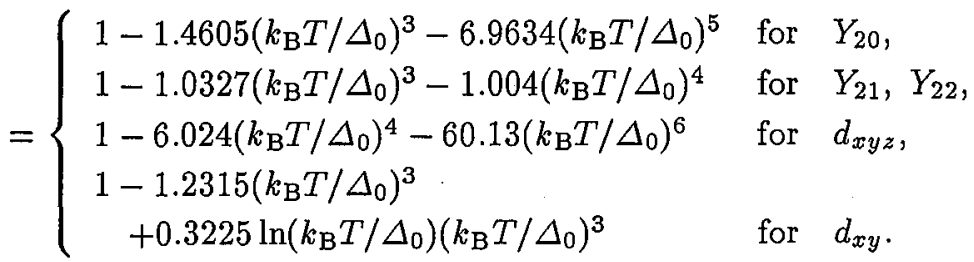

The temperature dependence of $\Delta / \Delta_{0}$ for all above cases is presented in Fig. 2b. For $S$-wave pairing $f(|D|)=\delta(|D|-1)$ so $f(|D|)=0$ for $|D|<1$. When all detailed calculations are carried out it turns out that $\Delta / \Delta_{0}=1$. In order to get well known formula for energy gap in the low temperature limit: $\Delta / \Delta_{0}=$ $1-\sqrt{2 \pi k_{\mathrm{B}} T / \Delta_{0}} \exp \left(-\Delta_{0} / k_{\mathrm{B}} T\right)$, more accurate approximation of $W(x) \approx \ln (x)-$ $2 \sqrt{\pi / 2 x} \exp (-2 x)$ for large $x$ should be taken into account. For $D$-wave pairing this correction is negligible.
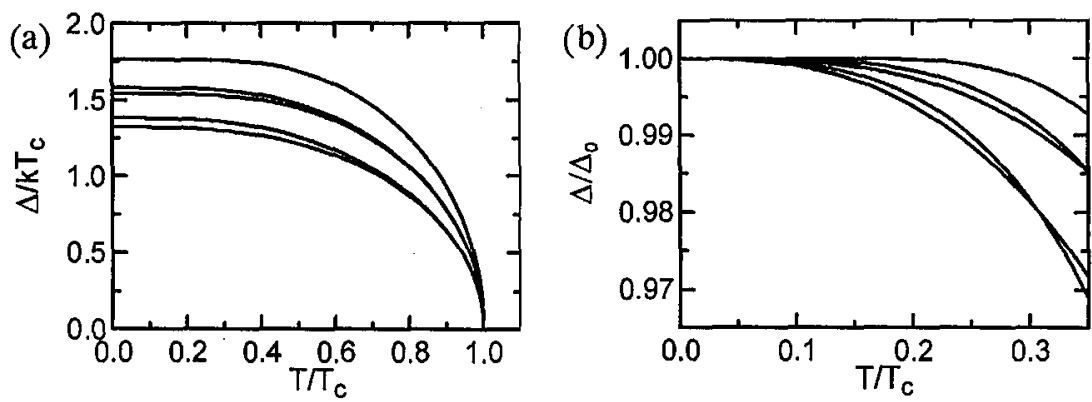

Fig. 2. The reduced average energy gap as a function of reduced temperature for $S$-wave and different $D$-wave pairing solutions obtained: (a) from numerical calculation for $Y_{00}, d_{x y z}, Y_{21}$ (or $Y_{22}$ ), $Y_{20}, d_{x y}$ from the top, respectively; and (b) analytically in limiting case $T \rightarrow T_{\mathrm{c}}$ for $Y_{00}, Y_{21}$ (or $Y_{22}$ ), $d_{x y z}, Y_{20}, d_{x y}$ from the top, respectively. The average energy gap for $D$-wave pairing in low temperature limit is changing according to power function (not exponentially like in $S$-wave pairing case). Note that there are no linear and square terms. 


\section{Thermodynamic potential and specific heat}

The thermodynamic potential difference between the paired and normal state, $\Omega_{\mathrm{s}}-\Omega_{\mathrm{n}}=\Delta \Omega$, is the function of temperature as well as pairing interaction. For purpose of calculating $\Delta \Omega$ one can use a method of "switching on" the interaction, which consists in calculating the variation of $\Delta \Omega$ during increasing interaction parameters from zero up to given values. According to [6] the differential $\mathrm{d} \Delta \Omega / \mathrm{d} g_{L}$ is equal to

$$
\begin{aligned}
\frac{\mathrm{d} \Delta \Omega}{\mathrm{d} g_{L}} & =(2 L+1) N(0)\left\langle\left\langle P_{L}\left(k k^{\prime}\right) \Delta_{k}^{*} \Phi_{k}\right\rangle_{k} \Delta_{\boldsymbol{k}^{\prime}} \Phi_{\boldsymbol{k}^{\prime}}\right\rangle_{\boldsymbol{k}^{\prime}} \\
& =N(0) \sum_{M}\left|\left\langle Y_{L M}^{*}(k) \Delta_{\boldsymbol{k}} \Phi_{\boldsymbol{k}}\right\rangle\right|^{2}=N(0) \frac{\Delta^{2}}{g_{L}^{2}} \sum_{M}\left|\Delta_{L M}\right|^{2} .
\end{aligned}
$$

It is very useful changing the highest parameter $g_{\max }$ keeping all values of $1 / g_{L}-1 / g_{\max }$ constant. In this case

$$
\frac{\mathrm{d} \Delta \Omega}{\mathrm{d} g_{\max }}=\sum_{L} \frac{\mathrm{d} \Delta \Omega}{\mathrm{d} g_{L}} \frac{\mathrm{d} g_{L}}{\mathrm{~d} g_{\max }}=N(0) \frac{\Delta^{2}}{g_{\max }^{2}} \sum_{L M}\left|\Delta_{L M}\right|^{2}=N(0) \frac{\Delta^{2}}{g_{\max }^{2}},
$$

where the formula ( 7 ) was used. Therefore the thermodynamic potential difference is equal to

$$
\begin{aligned}
\frac{\Delta \Omega}{N(0)} & =\int_{0}^{g_{\max }} \frac{\Delta^{2}}{g^{\prime 2}} \mathrm{~d} g^{\prime}=-\int_{1 / g_{\max }}^{\infty} \Delta^{2} \mathrm{~d} \frac{1}{g^{\prime}}=-\int_{1 / T_{\mathrm{c}}}^{\infty} \Delta^{2} \frac{\mathrm{d} 1 / g^{\prime}}{\mathrm{d} 1 / T_{\mathrm{c}}^{\prime}} \mathrm{d} \frac{1}{T_{\mathrm{c}}^{\prime}} \\
& =-\int_{1 / T_{\mathrm{c}}}^{\infty} \frac{\Delta^{2}}{1 / T_{\mathrm{c}}^{\prime}} \mathrm{d} \frac{1}{T_{\mathrm{c}}^{\prime}}
\end{aligned}
$$

where the order of integration was changed and used Eq. (12). Additionally, in this case, the relation between $\Delta / k_{\mathrm{B}} T_{\mathrm{c}}$ and $T / T_{\mathrm{c}}$, which depends just on values of $1 / g_{L}-1 / g_{\max }$ (see (13)), can be applied. Introducing the parameters $Y=\Delta / k_{\mathrm{B}} T_{\mathrm{c}}$ and $X=T / T_{\mathrm{c}}$ Eq. (29) can be put in the form

$$
\frac{\Delta \Omega}{N(0) k_{\mathrm{B}}^{2} T_{\mathrm{c}}^{2}}=-X^{2} \int_{X}^{1} \frac{Y^{2}\left(X^{\prime}\right)}{X^{\prime 3}} \mathrm{~d} X^{\prime} \text {. }
$$

The upper limit in the integral is limited to one because $Y(X>1)=0$. In turn, substituting (18) into (30) and using well-known relation

$$
\Delta C=-T \partial^{2} \Delta \Omega / \partial T^{2},
$$

where $\Delta C=C_{\mathrm{s}}-C_{\mathrm{n}}$ is the specific heat difference between the paired and normal state, one gets thermodynamic potential and specific heat in the limit $T \rightarrow T_{\mathrm{c}}$

$$
\frac{\Delta \Omega}{N(0) k_{\mathrm{B}}^{2} T_{\mathrm{c}}^{2}}=-\frac{4 \pi^{2}}{7 \zeta(3)\left\langle\left|D_{k}\right|^{4}\right\rangle} X^{2}=-\frac{X}{2} \frac{\Delta C}{N(0) k_{\mathrm{B}}^{2} T_{\mathrm{c}}} .
$$

According to [6] $C_{\mathrm{n}}=(2 / 3) \pi^{2} N(0) k_{\mathrm{B}}^{2} T$ so the reduced specific heat jump $\Delta C / C_{\mathrm{n}}$ for $T=T_{\mathrm{c}}$ is equal to 0.6655 for $Y_{20}, d_{x y}$ solutions, 0.998 for $Y_{2 \pm 1}, Y_{2 \pm 2}, d_{x y z}$ ones, and 1.426 in $S$-wave pairing case, respectively.

Because the function $Y^{2}(X)$ is known only in the limiting cases one cannot use Eq. (30) for calculation of thermodynamic potential for $T \rightarrow 0$. But when the 
term $Y^{2}(0) / X^{3}$ is taken out of the integral in (30), the integrated function will not have the singularity for $T=0$ anymore. Then the limit of integration can be changed

$$
\begin{gathered}
\frac{\Delta \Omega}{N(0) k_{\mathrm{B}}^{2} T_{\mathrm{c}}^{2}}=-\frac{Y^{2}(0)}{2}+\frac{X^{2} Y^{2}(0)}{2}-X^{2} \int_{X}^{1} \frac{Y^{2}\left(X^{\prime}\right)-Y^{2}(0)}{X^{\prime 3}} \mathrm{~d} X^{\prime} \\
=-\frac{Y^{2}(0)}{2}+X^{2} \int_{0}^{X} \frac{Y^{2}\left(X^{\prime}\right)-Y^{2}(0)}{X^{\prime 3}} \mathrm{~d} X^{\prime}+X^{2} \text { const. }
\end{gathered}
$$

It can be proved (see Appendix) that $X^{2}$ const $=(1 / 3) \pi^{2} X^{2}=-\Omega_{\mathrm{n}}(X)$, so substituting (26) into (33) and then using (31) one obtains

$$
\begin{aligned}
& \Omega_{\mathrm{s}} / N(0) k_{\mathrm{B}}^{2} T+\mathrm{c}^{2} \\
& = \begin{cases}-0.2901-0.6427\left(T / T_{\mathrm{c}}\right)^{2}-0.5352\left(T / T_{\mathrm{c}}\right)^{4} & \text { for } Y_{20}, \\
-0.3622-0.4067\left(T / T_{\mathrm{c}}\right)^{2}-0.1281\left(T / T_{\mathrm{c}}\right)^{3} & \text { for } Y_{21}, Y_{22}, \\
-0.3791-0.7341\left(T / T_{\mathrm{c}}\right)^{3}-1.469\left(T / T_{\mathrm{c}}\right)^{5} & \text { for } d_{x y z}, \\
-0.2665-0.755\left(T / T_{\mathrm{c}}\right)^{2}+0.148\left(T / T_{\mathrm{c}}\right)^{2} \ln \left(T / T_{\mathrm{c}}\right) & \text { for } d_{x y},\end{cases} \\
& C_{\mathrm{s}} / N(0) k_{\mathrm{B}}^{2} T_{\mathrm{c}}^{2} \\
& = \begin{cases}12.69\left(T / T_{\mathrm{c}}\right)^{2}+35.28\left(T / T_{\mathrm{c}}\right)^{4} & \text { for } Y_{20}, \\
8.032\left(T / T_{\mathrm{c}}\right)^{2}+5.061\left(T / T_{\mathrm{c}}\right)^{3}+7.151\left(T / T_{\mathrm{c}}\right)^{4} & \text { for } Y_{21}, Y_{22}, \\
29.01\left(T / T_{\mathrm{c}}\right)^{3}+145.3\left(T / T_{\mathrm{c}}\right)^{5} & \text { for } d_{x y z}, \\
10.13\left(T / T_{\mathrm{c}}\right)^{2}-4.215\left(T / T_{\mathrm{c}}\right)^{2} \ln \left(T / T_{\mathrm{c}}\right) & \text { for } d_{x y} .\end{cases}
\end{aligned}
$$

The thermodynamic potential and specific heat for $S$-wave paired system are given by the expressions (see [6]):

$$
\begin{aligned}
& \Omega_{\mathrm{s}} / N(0) k_{\mathrm{B}}^{2} T_{\mathrm{c}}^{2} \\
& \quad=-\left(\Delta_{0} / k_{\mathrm{B}} T_{\mathrm{c}}\right)^{2}\left[1 / 2+2 \sqrt{2 \pi}\left(\Delta_{0} / k_{\mathrm{B}} T\right)^{-1 / 2} \exp \left(-\Delta_{0} / k_{\mathrm{B}} T\right)\right] \\
& C_{\mathrm{s}} / N(0) k_{\mathrm{B}}^{2} T_{\mathrm{c}}=2 \sqrt{2 \pi}\left(\Delta_{0} / k_{\mathrm{B}} T_{\mathrm{c}}\right)\left(\Delta_{0} / k_{\mathrm{B}} T\right)^{3 / 2} \exp \left(-\Delta_{0} / k_{\mathrm{B}} T\right)
\end{aligned}
$$

The temperature dependence of thermodynamic potential and specific heat are shown in Fig. 3 and Fig. 4, respectively. To present the differences between the solutions more clearly, in Fig. $4\left[C / C_{\mathrm{n}}\left(T_{\mathrm{c}}\right)\right] /\left(T / T_{\mathrm{c}}\right)^{2}$ as the function of $T / T_{\mathrm{c}}$ is plotted. In these convenient units, in zero temperature limit, one gets constant dependencies for $Y_{20}, Y_{21}$, and $Y_{22}$, linear dependence for $d_{x y z}$, logarithmic one for $d_{x y}$, and exponential one for $S$-wave pairing $\left(Y_{00}\right)$ solution. 

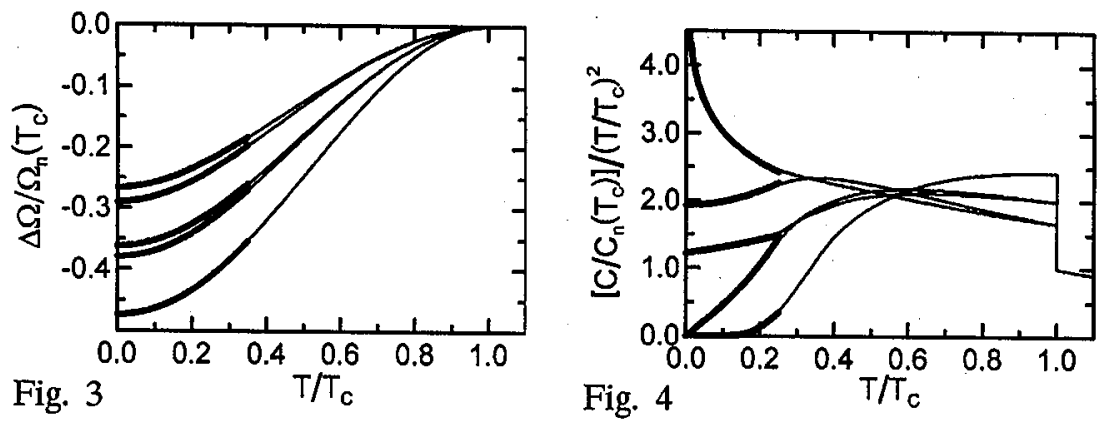

Fig. 3. The reduced thermodynamic potential difference between superconducting and normal state $\Delta \Omega / \Omega_{\mathrm{n}}\left(T_{\mathrm{c}}\right)$ as a function of reduced temperature for $S$-wave and different $D$-wave solutions obtained analytically in the limit $T \rightarrow T_{\mathrm{c}}$ for $d_{x y}, Y_{20}, Y_{21}$ (or $Y_{22}$ ), $d_{x y z}, Y_{00}$ (thick lines from the top respectively); and from numerical calculations (thin lines).

Fig. 4. The reduced specific heat $\left[C / C_{\mathrm{n}}\left(T_{c}\right)\right] /\left(T / T_{c}\right)^{2}$ as a function of reduced temperature for $S$-wave and different $D$-wave solutions obtained analytically in the limit $T \rightarrow T_{c}$ for $d_{x y}, Y_{20}, Y_{21}$ (or $Y_{22}$ ), $d_{x y z}, Y_{00}$ (thick lines from the top respectively); and from numerical calculations (thin lines). In zero temperature limit constant dependence for $Y_{20}, Y_{21}$, and $Y_{22}$ harmonics, linear one for $d_{x y z}$, logarithmic one for $d_{x y}$, and exponential one for $Y_{00}$ ( $S$-wave pairing solution) are obtained.

\section{Conclusions}

The obtained non-exponential temperature dependence of the energy gap value, the thermodynamic potential and the specific heat of $D$-wave paired system in low temperature limit arise from the nodes occurring on the Fermi surface i.e. places where energy gap vanishes. Let us emphasize that this feature remarkably distinguishes $D$-wave paired system from the $S$-wave paired one.

Moreover, according to the above results, the temperature dependence of the specific heat for possible $D$-wave solutions, represented by various shapes of energy gap, can be essentially different for particular cases. These qualitative discrepancies are directly connected with the form of density states function on the Fermi surface $(1 / 4 \pi) \partial \Omega / \partial|D|$ for small energy gap values.

Nevertheless, in isotropic system only solution with $d_{x y z}$ energy gap shape should be stable because it minimizes the thermodynamic potential.

Finally, it is worthy mentioning that there is no linear term in the specific heat in the limit $T \rightarrow 0$ for both $D$-wave and $S$-wave paired system.

\section{Appendix}

For calculating the terms of the thermodynamic potential expansion into series of $T / T_{\mathrm{c}}$ powers in zero temperature limit one can use the method presented in [6] adapted for $D$-wave paired system. As formerly (see (27), (29)):

$$
\frac{\Delta \Omega}{N(0)}=\int_{0}^{g_{2}} \frac{\Delta^{2}}{{g^{\prime}}^{2}} \mathrm{~d} g^{\prime}=\int_{\infty}^{1 / g_{2}} \Delta^{2} \mathrm{~d} \frac{1}{g^{\prime}}=\int_{0}^{\Delta} \Delta^{\prime 2} \frac{\mathrm{d}}{\mathrm{d} \Delta^{\prime}} \frac{1}{g_{2}} \mathrm{~d} \Delta^{\prime}
$$


After integrating by parts and substituting (16) and then (4):

$$
\begin{aligned}
\frac{\Delta \Omega}{N(0)} & =\frac{\Delta^{2}}{g_{2}}-2 \int_{0}^{\Delta} \Delta^{\prime}\left\langle\left|D_{k}\right|^{2} \Phi_{k}\right\rangle \mathrm{d} \Delta^{\prime} \\
& =\frac{\Delta^{2}}{g_{2}}-2 \int_{0}^{\hbar \omega}\left\langle\int_{0}^{\Delta_{k}} \Delta^{\prime} \frac{\tanh \left(E^{\prime} / 2 k_{\mathrm{B}} T\right)}{E^{\prime}} \mathrm{d} \Delta^{\prime}\right\rangle \mathrm{d} \xi,
\end{aligned}
$$

where $E^{\prime}=\left(\left|\Delta^{\prime}\right|^{2}+\xi^{2}\right)^{1 / 2}$. The last integral can be simplified changing the order of integration $\Delta^{\prime} \rightarrow E^{\prime}$ :

$$
\begin{aligned}
\frac{\Delta \Omega}{N(0)} & =\frac{\Delta^{2}}{g_{2}}-2 \int_{0}^{\hbar \omega}\left\langle\int_{0}^{E_{k}} \tanh \left(E^{\prime} / 2 k_{\mathrm{B}} T\right) \mathrm{d} E^{\prime}\right\rangle \mathrm{d} \xi \\
& =\frac{\Delta^{2}}{g_{2}}-4 k_{\mathrm{B}} T \int_{0}^{\hbar \omega}\left\langle\ln \frac{\cosh \left(E_{\boldsymbol{k}} / 2 k_{\mathrm{B}} T\right)}{\cosh \left(\xi / 2 k_{\mathrm{B}} T\right)}\right\rangle \mathrm{d} \xi \\
& =\frac{\Delta^{2}}{g_{2}}+4 k_{\mathrm{B}} T \int_{0}^{\hbar \omega}\left\{\ln \left[1+\exp \left(-\xi / k_{\mathrm{B}} T\right)\right]\right. \\
& -\frac{1}{2}\left\langle\frac{E_{\boldsymbol{k}}-\xi}{k_{\mathrm{B}} T}\right\rangle-\left\langle\ln \left[1+\exp \left(-E_{\boldsymbol{k}} / k_{\mathrm{B}} T\right)\right]\right\rangle \mathrm{d} \xi .
\end{aligned}
$$

Let us calculate the successive terms in the above formula separately. According to [6]

$$
\begin{aligned}
& 4 k_{\mathrm{B}} T \int_{0}^{\infty} \ln \left[1+\exp \left(-\xi / k_{\mathrm{B}} T\right)\right] \mathrm{d} \xi=\frac{1}{3} \pi^{2}\left(k_{\mathrm{B}} T\right)^{2} \approx-\Omega_{n}(T), \\
& -2 \int_{0}^{\hbar \omega}\left\langle E_{k}-\xi\right\rangle \mathrm{d} \xi \approx-\left\langle\frac{1}{2}\left|\Delta_{k}\right|^{2}+\left|\Delta_{k}\right|^{2} \ln \left(2 \hbar \omega /\left|\Delta_{k}\right|\right)\right\rangle \\
& =-\frac{\Delta^{2}}{2}-\frac{\Delta^{2}}{g_{L}}-\Delta^{2} \ln \frac{\Delta_{0}}{\Delta},
\end{aligned}
$$

where in the last equation the relation (7) and (16) with (9) and (11) is used. In the third term the integration over an angle $\Omega_{\boldsymbol{k}}$ can be replaced by the integration over value of $\left|D_{\boldsymbol{k}}\right|$, so

$$
\begin{aligned}
4 k_{\mathrm{B}} T & \left\langle\int_{0}^{\infty} \ln \left[1+\exp \left(-E_{\boldsymbol{k}} / k_{\mathrm{B}} T\right)\right] \mathrm{d} \xi\right\rangle \\
& =4\left(k_{\mathrm{B}} T\right)^{2} \int_{0}^{|D|_{\max }} f(|D|) U(\tau|D|) \mathrm{d}|D|,
\end{aligned}
$$

where $U(\tau|D|)=2 \int_{0}^{\infty} \ln \left[1+\exp \left(-2 \sqrt{u^{2}+\tau^{2}|D|^{2}}\right)\right] \mathrm{d} u, \tau=\Delta / 2 k_{\mathrm{B}} T$ and $u=$ $\xi / 2 k_{\mathrm{B}} T$.

Note that the upper limit in the integrals in Eqs. (41) and (43) were expanded up to infinity because of their quick convergence. For the same reason only a part 
with small $\tau|D|$, i.e. $\tau|D|<10$, is significant. For low temperatures $\tau$ is large, therefore $|D|$ in significant region is always small and this allows to approximate $f(|D|)$ by polynomial

$$
\begin{gathered}
\int_{0}^{|D|_{\max }} f(|D|) U(\tau|D|) \mathrm{d}|D| \approx \int_{0}^{10 / \tau} \sum_{n} A_{n}|D|^{n} U(\tau|D|) \mathrm{d}|D| \\
=\sum_{n} \frac{A_{n}}{\tau^{n+1}} \int_{0}^{10} x^{n} U(x) \mathrm{d} x=\sum_{n} \frac{A_{n} H_{n}}{\tau^{n+1}}
\end{gathered}
$$

or logarithmic function

$$
\begin{aligned}
\int_{0}^{|D|_{\max }} & f(|D|) U(\tau|D|) \mathrm{d}|D| \approx \int_{0}^{10 / \tau}[E \ln (|D|)+F] U(\tau|D|) \mathrm{d}|D| \\
= & \frac{F-E \ln (\tau)}{\tau} \int_{0}^{10} U(x) \mathrm{d} x+\frac{E}{\tau} \int_{0}^{10} \ln (x) U(x) \mathrm{d} x \\
= & \frac{[F-E \ln (\tau)] H_{0}}{\tau}+\frac{E I}{\tau},
\end{aligned}
$$

where $H_{n}=\int_{0}^{10} x^{n} U(x) \mathrm{d} x$. Hence $H_{0}=0.7081, H_{1}=0.4735, H_{2}=0.5726$, $H_{3}=0.9855$, and $I=\int_{0}^{10} \ln (x) U(x) \mathrm{d} x=-0.6353$. Finally collecting together the obtained results

$$
\frac{\Delta \Omega}{N(0)}=-\frac{\Delta^{2}}{2}-\Delta^{2} \ln \frac{\Delta_{0}}{\Delta}-4\left(k_{\mathrm{B}} T\right)^{2} \sum_{n} \frac{A_{n} H_{n}}{\tau^{n+1}}+\frac{1}{3} \pi^{2}\left(k_{\mathrm{B}} T\right)^{2}
$$

or

$$
\begin{aligned}
& \frac{\Delta \Omega}{N(0)}=-\frac{\Delta^{2}}{2}-\Delta^{2} \ln \frac{\Delta_{0}}{\Delta}-4\left(k_{\mathrm{B}} T\right)^{2}\left\{\frac{[F-E \ln (\tau)] H_{0}}{\tau}+\frac{E I}{\tau}\right\} \\
& +\frac{1}{3} \pi^{2}\left(k_{\mathrm{B}} T\right)^{2} .
\end{aligned}
$$

Substituting $\tau=\Delta / 2 k_{\mathrm{B}} T$, and $\Delta$ from (26) as well as using approximation $\ln (x) \approx$ $x-1$, one may see that the constant term is equal to $\Delta_{0} / 2$. There is no linear term with respect to $T$, and the square term is equal to $(1 / 3) \pi^{2}$.

\section{References}

[1] D. Scalapino, Phys. Rep. 250, 329 (1995).

[2] M. Sigrist, K. Ueda, Rev. Mod. Phys. 63, 239 (1991).

[3] W. Kumala, R. Gonczarek, Acta Phys. Pol. A 93, 539 (1998).

[4] R. Gonczarek, M. Mulak, Phys. Lett. A 251, 262 (1999).

[5] M. Mulak, R. Gonczarek, Acta Phys. Pol. A 92, 1177 (1997).

[6] A.L. Fetter, J.D. Walecka, Quantum Theory of Many-Particle Systems, McGraw-Hill, New York 1971, Sec. 51.

[7] P.W. Anderson, W.F. Brinkman, in: The Physics of Liquid and Solid Helium, Eds. K.H. Bennemann, J.B. Ketterson, Part II, Wiley, New York 1978, p. 208.

[8] P.W. Anderson, P. Morel, Phys. Rev. 123, 1911 (1961). 